\title{
Does Mesh Augmentation of the Hiatal Repair with Gore Bio-A® Improve Symptomatic Outcomes in Laparoscopic Fundoplication?
}

\author{
Christodoulidou Ma , Kosai NR ${ }^{\mathrm{b}}$, Rajan R ${ }^{\mathrm{b}}$, Hassan $\mathrm{S}^{\mathrm{a}}$, Das $\mathrm{S}^{\mathrm{c}}$, Sutton PAd, Varghese Ja \\ ${ }^{a}$ General Surgery Department, Royal Bolton Hospital NHS Foundation Trust, Minerva Road, Bolton BL4 OJR \\ ${ }^{b}$ Department of Surgery, Universiti Kebangsaan Malaysia Medical Center (UKMMC), Kuala Lumpur, Malaysia \\ 'Department of Anatomy, Universiti Kebangsaan Malaysia Medical Center (UKMMC), Kuala Lumpur, Malaysia \\ dUniversity of Liverpool, Institute of Translational Medicine, Liverpool, United Kingdom
}

\begin{abstract}
Introduction: Laparoscopic fundoplication is performed for the management of symptomatic hiatus hernias and gastro-oesophageal reflux disease (GORD) refractory to medical therapy. We adopted the use of Gore Bio-A $₫$ for selected laparoscopic hiatus hernia repairs in 2011 and with this case series aimed to establish whether mesh augmentation affects symptomatic outcomes. Methods: A retrospective review of prospectively collected data from all laparoscopic fundoplications performed by a single surgeon between October 2011 and January 2013 was performed. Patient specific data were entered into a proforma and analysed using Microsoft Excel ${ }^{\mathrm{TM}}$. Patient reported outcomes were assessed with a system specific quality of life questionnaire (GORD-HRQL) both pre and post-operatively. Results: Twenty-three patients underwent laparoscopic fundoplication during the study period. Gore Bio-A® re-enforcement of the hiatal repair was used in 14 patients and was the preferred option for those with pre-operative evidence of a large hiatus hernia. Whilst overall there was a statistically significant difference between pre and post-operative scores ( 21 vs $0, p=<0.0001$, Mann-Whitney $U$ test), there was no clear difference observed in pre-operative scores ( 22 vs $20, p=0.21$, Mann-Whitney $U$ test), postoperative scores ( 0 vs $0, p=0.92$, Mann-Whitney $U$ test) or symptom improvement ( 21 vs $20, p=0.24$, MannWhitney $\mathrm{U}$ test) between the mesh and non-mesh groups. Conclusions: Augmentation of the hiatal repair with biosynthetic mesh is safe, feasible and may contribute to improved symptomatic outcomes in selected cases with a large hiatus hernia. We suggest a further assessment with a larger randomised sample and long term follow-up for definitive evaluation.
\end{abstract}

KEYWORDS: Laparoscopic Fundoplication, Hiatus Hernia Repair, Gastro-oesophageal reflux disease, Biomesh

\section{INTRODUCTION}

Laparoscopic fundoplication has been used as the primary surgical treatment for large hiatal hernias and severe gastro-oesophageal reflux disease (GORD) refractory to medical therapy for many years, with various advancements in technique. ${ }^{1}$ Traditionally, these hernias were repaired by thoracotomy or laparotomy with a morbidity rate of $20 \%$ and mortality rate of $2 \%{ }^{2}$ Laparoscopy has significantly reduced morbidity. However, there is no study that has proven a reduction in recurrence rate when compared to traditional

Corresponding author:

Assoc. Prof. Dr. Nik Ritza Kosai

Head of Unit and Senior Consultant Surgeon, Minimally invasive, Upper Gastrointestinal and Bariatric Surgery Unit, Department of Surgery,

Universiti Kebangsaan Malaysia Medical Centre, Jalan Yaacob Latif, Bandar Tun Razak,

56000 Kuala Lumpur, Malaysia

Tel: +6-03-91456202 laparotomy/thoracotomy. Recurrence rates have been reported from $12 \%$ to as high as $42 \%$, following laparoscopic repair. ${ }^{3,4}$ Primary suture cruroplasty of hiatal hernias has been heavily criticised in recent years and high recurrence rates have led to the use of mesh reinforcement. Two randomised controlled trials have demonstrated a significant reduction in recurrence rates when using synthetic mesh as opposed to primary closure with sutures alone. ${ }^{5,6}$ However, this is not without its risks. The main drawbacks of prosthetic mesh are risk of erosion, stricture, ulceration and post-operative dysphagia.

More recently, the prospect of biologic mesh has been introduced in the place of prosthetic mesh. A large multicentre randomised controlled trial in 2006 demonstrated a statistically significant reduction in all measured symptoms following biomesh repair with SIS (porcine small intestinal mucosa), including heartburn, regurgitation, postprandial pain, early satiety, chest pain and dysphagia at 6 months. There was also a 2.5 -fold reduction in the number of true recurrences (measured as a hernia $>2 \mathrm{~cm}$ ) found on endoscopic 
examination at 6 months. ${ }^{7}$

Gore Bio-A $\AA$ is a biological mesh (Fig.1) consisting of a unique polyglycolic acid and trimethylene carbonate absorbable synthetic polymer. It has an open matrix structure that acts as a scaffold for native tissue in-growth. Small studies have shown satisfactory short-term results with low recurrence rates when using Gore Bio- $A \AA$.8,9 Within our trust, we have adopted the use of Gore Bio- $A \circledast$ for selected laparoscopic hiatal hernia repairs. In this retrospective study, we review the short-term outcome of patients who have undergone laparoscopic fundoplication in our hospital following the introduction of Gore Bio-A®. Specifically, we set out to identify whether or not mesh augmentation of the hiatal repair has any effect on symptomatic outcomes, as well as complications, postoperative length of stay and the need for further intervention.

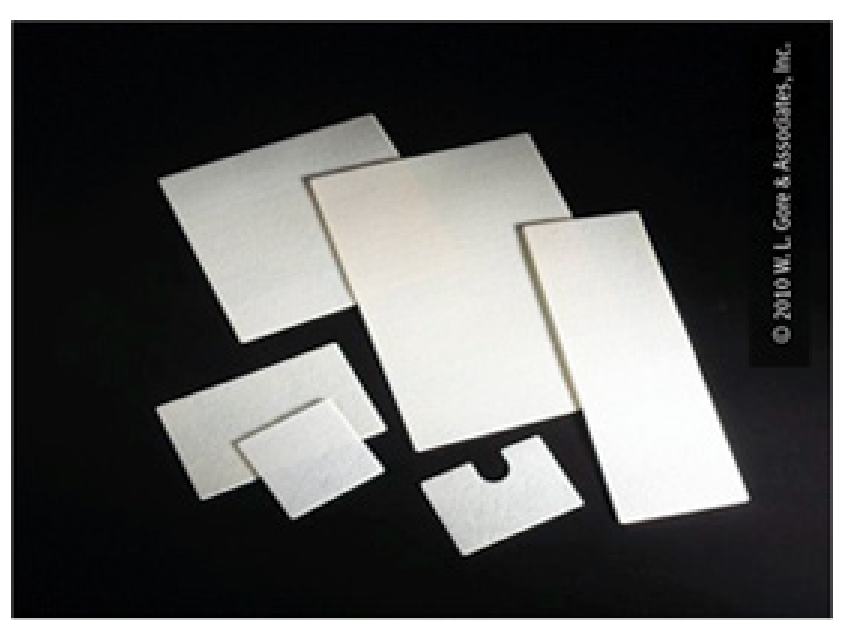

Figure 1. Gore Bio- $A ®$, samples of types of biomesh

\section{MATERIALS AND METHODS}

We performed a retrospective review of a prospectively maintained dataset spanning a 16 month period from October 2011 to January 2013 of all patients undergoing laparoscopic fundoplication. This time period was selected as the most recent dataset available following the introduction of Gore Bio- $A \AA$ in our trust. Data pertaining to patient demographics, preoperative investigations, intra-operative events, follow-up and outcomes were extracted from these records and entered into a proforma designed specifically for use within this study. These were subsequently entered into a Microsoft Excel $^{T M}$ spreadsheet for analysis. In addition, a GORD specific quality of life questionnaire was sent out to all study participants to record symptomatology both pre and post operatively on a 5-point scale. ${ }^{1}$ (Fig. 2 ).

All patients undergoing surgery for laparoscopic fundoplication were assessed pre-operatively by the admitting team. The criteria for listing included:

1. Patients with severe symptoms of GORD (refractory to medical therapy) with evidence of reflux on oesophago-gastro-duodenoscopy (OGD) or barium swallow.

2. The presence of a large symptomatic hiatus hernia.

3. A 24hr pH-manometry indicative of reflux in symptomatic patients with a significant history of GORD and no evidence of GORD on OGD or barium swallow.

All patients underwent an anaesthetic assessment preoperatively. Operations were performed by a single surgeon using the same surgical approach. Where present, the hiatus hernia was reduced and sutures applied to the posterior and anterior crus and the angle of His. All patients had an intra-corporeally sutured anterior wrap. Those with large hiatus hernias $(>5 \mathrm{~cm})$ received mesh re-enforcement which again was secured with sutures.

Post-operatively, all patients were immediately put on a soft diet and were monitored for dysphagia. Once patients were fit for discharge and able to tolerate diet satisfactorily, a plan for outpatient follow up was implemented. If any immediate, intermediate or late post-operative complications occurred, these were approached and dealt with by the same team. Patients were routinely followed up at 6 months to assess symptom outcomes and post-operative recovery. A subjective symptomatic approach was used to assess procedural success and post-operative investigations were reserved for patients with complications or recurrence of their symptoms. Patients were discharged back to primary care one year following the operation providing there were no ongoing management issues.

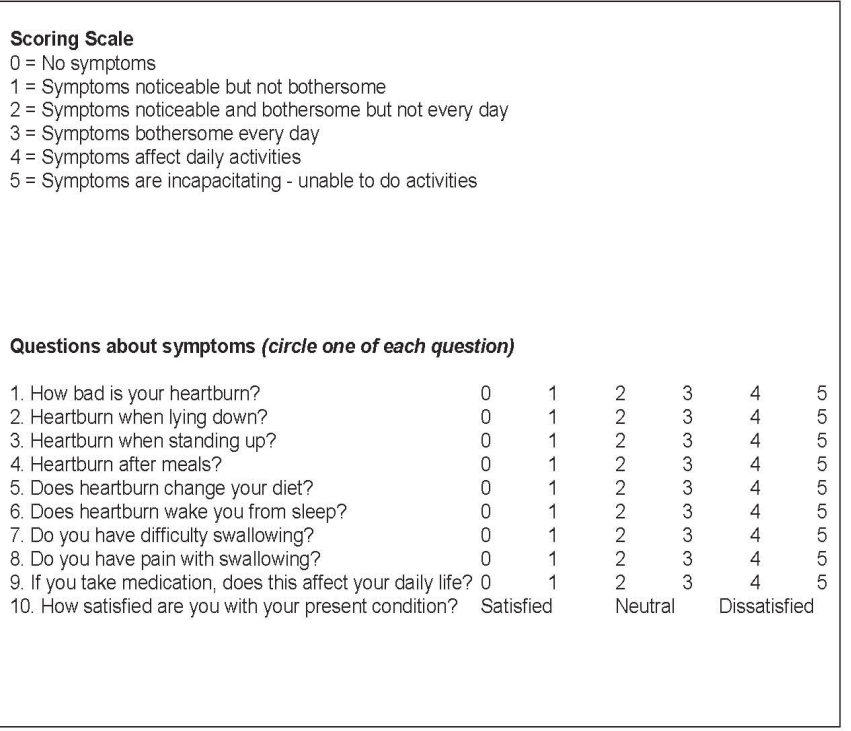

Figure 2. GORD-HRQL, a validated QOL score for patients suffering from GORD, Was utilised to assess symptomatology related $\mathrm{QOL}$ in this study 


\section{RESULTS}

\section{Patient Population}

Twenty-three patients were identified, 14 (61\%) of which were males with a median (IQR) age of 60 (30-82) years. The median (IQR) body mass index (BMI) of all patients was 26.6 (24.5-31.4), which was slightly higher in the female population (27.8 vs. 26.4). The most commonly documented co-morbidities were: obesity $(35 \%)$, alcohol excess $(22 \%)$, smoking [including ex-smokers] (22\%), hypertension (17\%), chronic obstructive pulmonary disease $(13 \%)$ and asthma (9\%). A history of stroke, renal impairment and diabetes mellitus were each present in $4 \%$ of patients. Nine patients $(39 \%)$ in this study had previous abdominal or pelvic surgery, specifically: appendicectomy $(13 \%)$, laparotomy $(9 \%)$, laparoscopic cholecystectomy (4\%), open cholecystectomy (4\%), epigastric hernia repair (4\%) and diagnostic laparoscopy $(4 \%)$.

Presenting symptoms and Pre-operative investigations Presenting symptoms are documented in Table 1 , with 15 patients $(65 \%)$ presenting with multiple symptomatology. The most commonly presenting symptoms were GORD (70\%) and epigastric pain (52\%). The reported median length of time with symptoms was 3 years (range 1 to 17). All patients had an OGD to investigate their symptoms prior to operative intervention. Twenty patients $(87 \%)$ were reported to have evidence of hiatus hernia on OGD and in 14 cases $(70 \%)$ this was large $(>5 \mathrm{~cm})$ or intrathoracic. Eight patients $(35 \%)$ had endoscopic evidence of reflux oesophagitis and four patients (17\%) had Barrett's oesophagus (length $1-2 \mathrm{~cm})$.

Barium swallow was not performed routinely as a first line investigation for GORD in our trust. It was performed in 8 patients in this study (35\%) where OGD was unable to provide an explanation for the patient's symptoms. Barium swallow demonstrated a hiatus hernia in 6 of these patients (75\%) and GORD in 3 patients $(37 \%)$. Interestingly, in one case the patient's OGD was reported as normal but the barium swallow revealed evidence of a large rolling hiatus hernia. Only 3 patients were referred for $24 \mathrm{hr} \mathrm{pH}$ manometry. Two of these patients had a normal OGD, and one showed evidence of a small hiatus hernia. All 3 had evidence of reflux during this test with a DeMeester score of 22.8-56.6. In one of these cases there was a total of 339 reflux episodes despite a normal OGD.

Table 1: Presenting symptoms reported by our patient population.

\begin{tabular}{|l|l|l|}
\hline Symptom & $\begin{array}{l}\text { Number of patients } \\
(\mathbf{\%})\end{array}$ & Comments \\
\hline $\begin{array}{l}\text { Gastro-esophageal } \\
\text { reflux disease }\end{array}$ & $16(70)$ & Refractory to medical therapy \\
\hline Epigastric pain & $12(52)$ & $\begin{array}{l}\text { Secondary to large hiatus hernia } \\
(>5 \mathrm{~cm} \text { or intrathoracic })\end{array}$ \\
\hline Regurgitation & $9(39)$ & All types \\
\hline Gastrointestinal bleed & $3(13)$ & $\begin{array}{l}\text { Secondary to peptic ulcer disease, } \\
\text { Helicobacter pylori -ve in last } \\
\text { OGD and no active PUD in any of } \\
\text { three cases pre-operatively }\end{array}$ \\
\hline $\begin{array}{l}\text { Gastric outlet } \\
\text { obstruction }\end{array}$ & $2(9)$ & $\begin{array}{l}\text { Found to have gastric volvulus } \\
\text { and intrathoracic hiatus hernia }\end{array}$ \\
\hline
\end{tabular}

\section{Intra-operative findings and procedures}

All operations were performed as previously described. Gore Bio-A® re-enforcement was used in 14 patients, the decision for which was made by the operating surgeon. Mesh re-enforcement was performed for large hiatus hernias $(>5 \mathrm{~cm})$ in 10 patients with documented evidence at OGD. One patient was identified at barium meal as having an intrathoracic hiatus hernia with volvulus despite a normal OGD, which was later confirmed intra-operatively. The remaining 3 patients who received mesh all had small-medium sized hiatus hernias but suffered from extremely severe GORD refractory to medical therapy (Table 2 ). Four of the 14 patients with an endoscopic documentation of a large hiatus hernia did not receive mesh, either due to significant adhesions or the intra-operative finding of a smaller than expected hiatus hernia. One patient (4\%) was converted to an open procedure due to adhesions and distorted anatomy resulting from a previous laparotomy for diaphragmatic rupture. There were no reported intraoperative complications or significant blood loss (defined as a loss of blood volume requiring transfusion) in any of the 23 cases.

Post-operative morbidity and mortality

The median length of stay was 1 day in the mesh group (range 1-38 days) and 2 days in the non-mesh group (range 1-6 days). In those individuals who received mesh, the incidence of post-operative dysphagia was $14 \%$ ( 2 patients) compared to $11 \%$ (1 patient) in the cohort who did not receive mesh $(p=0.83$, Chisquared). All three patients required subsequent OGD and dilatation. There was one minor surgical site infection in the mesh group which was successfully treated with a short course of oral antibiotics. One patient in each group developed hospital acquired 
Table 2: Demographics, presentation and pre-operative investigation findings in those patients undergoing mesh augmentation of the hiatal repair with Gore Bio-A®.

\begin{tabular}{|c|c|c|c|c|c|c|}
\hline $\begin{array}{l}\text { Patient } \\
\text { ID }\end{array}$ & Gender & Age & BMI & $\begin{array}{l}\text { Co- } \\
\text { morbidities }\end{array}$ & Presenting complaint & \begin{tabular}{|l|} 
Findings from pre- \\
operative investigations
\end{tabular} \\
\hline 1 & $\mathrm{~F}$ & 61 & 23.3 & None & Regurgitation & Large hiatus hernia (OGD) \\
\hline 2 & $\mathrm{~F}$ & 40 & 34.3 & $\begin{array}{l}\text { High alcohol } \\
\text { intake }\end{array}$ & $\begin{array}{l}\text { Reflux not controlled with } \\
\text { PPI }\end{array}$ & Large hiatus hernia (OGD) \\
\hline 3 & $\mathrm{M}$ & 67 & 31.6 & $\begin{array}{l}\text { Cerebro- } \\
\text { vascular } \\
\text { accident }\end{array}$ & $\begin{array}{l}\text { Regurgitation and reflux } \\
\text { not controlled with PPI }\end{array}$ & Large hiatus hernia (OGD) \\
\hline 4 & $\mathrm{~F}$ & 57 & 31.0 & Smoker & $\begin{array}{l}\text { Upper gastrointestinal } \\
\text { bleed and epigastric pain }\end{array}$ & Large hiatus hernia (OGD) \\
\hline 5 & $M$ & 70 & 26.9 & None & $\begin{array}{l}\text { Regurgitation and reflux } \\
\text { not controlled with PPI } \\
\text { and epigastric pain }\end{array}$ & $\begin{array}{l}\text { Large hiatus hernia found on } \\
\text { barium meal, OGD normal }\end{array}$ \\
\hline 6 & $\mathrm{~F}$ & 63 & 25.5 & None & $\begin{array}{l}\text { Reflux not controlled with } \\
\text { PPI }\end{array}$ & $\begin{array}{l}\text { Large hiatus hernia and } \\
\text { oesophagitis (OGD) }\end{array}$ \\
\hline 7 & $M$ & 82 & 24.3 & None & $\begin{array}{l}\text { Regurgitation and } \\
\text { epigastric pain }\end{array}$ & Large hiatus hernia (OGD) \\
\hline 8 & $M$ & 46 & 24.5 & None & $\begin{array}{l}\text { Reflux not controlled with } \\
\text { PPI and epigastric pain }\end{array}$ & $\begin{array}{l}\text { Large hiatus hernia and } \\
\text { oesophagitis (OGD) }\end{array}$ \\
\hline 9 & $\mathrm{~F}$ & 67 & 20.3 & Smoker & Epigastric pain & Large hiatus hernia (OGD) \\
\hline 10 & $\mathrm{~F}$ & 81 & 26.6 & Hypertension & $\begin{array}{l}\text { Regurgitation and } \\
\text { epigastric pain }\end{array}$ & $\begin{array}{l}\text { Large Hiatus Hernia and } \\
\text { oesophagitis and gastric } \\
\text { volvulus (OGD) }\end{array}$ \\
\hline 11 & $M$ & 65 & 26.0 & $\begin{array}{l}\text { COPD, } \\
\text { hypertension, } \\
\text { smoker, high } \\
\text { alcohol intake }\end{array}$ & $\begin{array}{l}\text { Regurgitation and reflux } \\
\text { not controlled with PPI } \\
\text { and epigastric pain }\end{array}$ & Small hiatus hernia (OGD) \\
\hline 12 & $M$ & 54 & 23.1 & $\begin{array}{l}\text { High alcohol } \\
\text { intake }\end{array}$ & $\begin{array}{l}\text { Reflux not controlled with } \\
\text { PPI }\end{array}$ & \begin{tabular}{|l|} 
Medium hiatus hernia and \\
Barrett's oesophagus (OGD)
\end{tabular} \\
\hline 13 & $M$ & 30 & 31.4 & Asthma & $\begin{array}{l}\text { Reflux not controlled with } \\
\text { PPI }\end{array}$ & $\begin{array}{l}\text { Small hiatus hernia (OGD), } \\
24 \mathrm{hr} \mathrm{pH} \text { manometry } \\
\text { indicative of reflux }\end{array}$ \\
\hline 14 & $\mathrm{~F}$ & 76 & 37.9 & $\begin{array}{l}\text { Hypertension, } \\
\text { high alcohol } \\
\text { intake }\end{array}$ & $\begin{array}{l}\text { Reflux not controlled with } \\
\text { PPI and epigastric pain }\end{array}$ & Large hiatus hernia (OGD) \\
\hline
\end{tabular}

\section{Outcomes}

An 82-year-old female who had a laparoscopic fundoplication for gastric volvulus and outlet obstruction sadly died on admission to the Accident and Emergency Department, 2 months following her surgery, the cause of which was identified as myocardial infarction. The remaining 22 patients were followed up for a minimum of 6 months. At first follow-up, 21 patients had obtained a degree of symptomatic relief with one patient complaining of occasional dysphagia to dry food. All 22 patients completed the GORD-HRQL questionnaire to allow analysis of their pre- and postoperative symptoms. Each variable stated in Figure 2 was scored from 0 to 5 with a total score of 45 in the most symptomatic patients. In the mesh group, the median pre-operative score was 22 out of 45 (range 0 to 42 ) and median post-operative score was 0 out of 45 (range 0 to 4 ). A total of $86 \%$ of patients reported a 'satisfactory' outcome following the operation. In the non-mesh group, the median preoperative symptomatic score was 20 out of 45 (range 0 to 31 ) and median post-operative score was 0 (range 0 to 3). A total of $89 \%$ of these patients reported symptomatic improvement. Whilst overall there was a statistically significant difference between pre and post-operative scores ( 21 vs $0, p=<0.0001$, MannWhitney $U$ test), there was no clear difference observed in pre-operative scores ( 22 vs $20, p=0.21$, Mann-Whitney $U$ test), post-operative scores ( 0 vs $0, p=0.92$, Mann-Whitney $U$ test) or symptom improvement ( 21 vs $20, p=0.24$, Mann-Whitney $U$ test) between the mesh and non-mesh groups.

The patient who underwent an open fundoplication re-presented 6 months following his procedure with adhesional small bowel obstruction requiring a laparotomy, small bowel resection and primary anastomosis. He made a full recovery and was discharged back to his home. No other late complications were observed.

\section{DISCUSSION}

Large hiatal hernias have always been a challenging entity for practicing surgeons and the standard of care for repair remains controversial. The literature suggests anything up to a $42 \%$ recurrence rate following laparoscopic repair of hiatal hernias, however further studies have shown a statistically 
significant improvement with the use of mesh reenforcement [10]. Studies measuring outcomes following augmentation with Gore Bio- $A \circledast$ remain limited, however small studies have shown a low recurrence rate of 5-9\% with no mesh-related complications such as erosion or dysphagia. ${ }^{8,9}$

Outcomes from laparoscopic fundoplication can be measured both objectively and subjectively but one could argue that if a patient's symptoms are improved, the procedure is successful. One study, based on symptomatic control, subjectively measured the success rate of open versus laparoscopic repair and reported a higher incidence of recurrence following open surgery. ${ }^{11}$ However, a case series which objectively measured recurrence by radiography or endoscopy, found true recurrence rates to be anything between $12-42 \%$ in the case of laparoscopic repair. ${ }^{3,4}$ In the present study, we measured outcomes subjectively using the patients' symptoms pre- and post-operatively in both the mesh and non-mesh groups. If symptoms suggested recurrence, the patient was followed up with an investigation such as a barium swallow or OGD. The advantage of this is the avoidance of unnecessary radiation or invasive intervention. Our study found that comparable outcomes were achieved in those undergoing augmentation of the hiatal repair with Gore Bio-A $₫$ compared to those who did not. This is clinically very significant, considering the fact that the patients receiving mesh augmentation represent the more severe end of the spectrum of pathology encountered.

In terms of complications postoperative dysphagia rates were higher in the group of patients who underwent mesh repair, although this did not reach statistical significance $(p=0.83)$. No recurrences were reported within this cohort, representing better outcomes than those previously published although our sample size is small. It has been shown that patients with hiatal hernia have ultra-structural abnormalities of the crural muscle tissue that was not present in patients with a normal gastroesophageal junction, which may explain the lower recurrence rates which have been reported with the use of mesh. ${ }^{7}$

Evidently, mesh repair is not without its complications. These include the risk of mesh erosion, ulceration and dysphagia. ${ }^{7}$ Although these risks are rare, they can cause significant morbidity. It is questionable as to whether these complications occur as frequently with biological mesh as with prosthetic mesh, particularly in the long term. A retrospective review of patients undergoing paraoesophageal hiatus hernia repair in 2011 reported no increase in the incidence of longterm dysphagia in patients undergoing biological mesh repair compared to a non-mesh repair. ${ }^{12}$ However, considering our study, it did report that immediate dysphagia is more commonly a problem following repair with a biological mesh when compared to a non-mesh repair. The study also states that this usually settles within a year, and therefore longer periods of follow-up are required. One possible explanation for this is that following the reabsorption of the bio- logical mesh by the body it is less likely to cause strictures or ulcerations when compared with prosthetic mesh. In view of this however, one would question the long-term success of biomesh once it has been fully reabsorbed. Oelschlager et al looked into this by extension of the 2006 multicentre randomised controlled trial where the same patients were followed up at 5 years post laparoscopic mesh repair of a hiatal hernia. Seventy-two of 108 patients were reassessed, 60 of which underwent OGD in addition to symptom reporting. The study concluded that there were no reports of any strictures, erosion or dysphagia with biomesh, however any conferred benefits, measured both subjectively or objectively, were diminished at five years. ${ }^{13}$

\section{CONCLUSION}

Our study demonstrated a slight increase in patient satisfaction with mesh reinforcement in laparoscopic fundoplication, with no real increase in complication rate. Furthermore, an equivalent improvement in symptom scores was seen between the mesh and nonmesh groups, significant given the more pronounced pathology present in the mesh group. Limitations of the study include the retrospective nature of the study, small sample size and lack of randomisation. Additionally, as this study represents a single surgeon's series an evolution in technique may have contributed to any observed differences. We conclude that the use of Gore Bio-A®to augment the hiatal repair in laparoscopic fundoplication is safe, feasible and may contribute to improved symptomatic outcomes in selected cases. Further evaluation in a larger randomised sample with long term follow-up is necessary for a full and complete evaluation of this technique.

\section{Acknowledgements}

With thanks to Gore ${ }^{\circledR}$ for supplying us with images of the biomesh.

\section{REFERENCES}

1. Velanovich V. The Development of the GERDHRQL symptom severity instrument. Dis Esophagus 2007; 20:130-4.

2. Ellis FH, Crozier RE, Shea JA. Paraoesophageal hiatus hernia. Arch Surg 1986; 121:416-420.

3. Horgan S, Eubanks TR, Jacobsen G, et al. Repair of paraesophageal hernias. Am J Surg 1999; 177:354-8

4. Hashemi M, Peters JH, DeMeester TR, et al. Laparoscopic repair of large type III hiatal hernia:objective follow up reveals high recurrence rate. J Am Coll Surg 2000; 190:554-61.

5. Frantizides CT, Madan AK, Carlson MA, et al. A prospective randomized trial of laparoscopic polytetrafluoroethylene (PTFE) patch repair vs simple cruroplasty for large hiatal hernia. Arch 
Surg 2002; 137:649-52.

6. Granderath FA, Schweiger UM, Kamolz T, et al. Laparoscopic Nissen fundoplication with prosthetic hiatal closure reduces postoperative intrathoracic wrap herniation: preliminary results of a prospective randomized functional and clinical study. Arch Surg 2005; 140:40-8.

7. Oelschlager BK, Pellegrini CA, Hunter J, et al. Biologic prosthesis Reduces Recurrence after laparoscopic paraesophageal hernia repair. Annals of Surgery 2006; 244:481-8.

8. Massullo JM, Singh TP, Dunnican WJ, et al. Preliminary Study of hiatal hernia repair using polyglycolic acid: Trimethylene Carbonate Mesh. JSLS 2012; 16:55-9.

9. Maheswaran IA, Conway A, Jethwa P. Laparoscopic repair of large and recurrent hiatus hernia with gore bio a mesh: interim experience. SAGES Abstract Archives 2013; unpublished.

10. Frantzides $C T$, Carlson MA, Loizides $S$, et al. Hiatal hernia repair with mesh: a survery of SAGES members. Surg Endosc 2010; 24:1017-24.

11. Schauer PR, Ikramuddin S, McLaughlin MD, et al. Comparison of laparoscopic versus open repair of paraesophageal hernia. Am J Surg 1998; 176: 659-65.

12. Goers TA, Cassera MA, Dunst CM, et al. Paraesophageal hernia repair with biomesh does not increase postoperative dysphagia. J Gastrointest Surg 2011; 15:1743-9.

13. Oelschlager BK, Pellegrini CA, Hunter J, et al. Biologic Prosthesis to prevent recurrence after laparoscopic paraesophageal hernia repair: Long term follow up from a multicenter, prospective, randomized trial. J Am Coll Surg 2011; 213:461-8. 